\title{
The relationship between the carotid canal and mandibular condyle: an anatomical study with application to surgical approaches to the skull base via the infratemporal fossa
}

\author{
Fernando Alonso ${ }^{1}$, Shenell Bernard ${ }^{2}$, Paul A. Irwin ${ }^{1}$, R. Isaiah Tubbs ${ }^{1}$, Joe Iwanaga ${ }^{1}$, Marios Loukas ${ }^{2}$, \\ Rod J. Oskouian $^{1,3}$, R. Shane Tubbs ${ }^{1}$ \\ ${ }^{1}$ Seattle Science Foundation, Seattle, Washington, USA \\ ${ }^{2}$ Faculty of Medicine, St. George's University, Grenada, West Indies \\ ${ }^{3}$ Swedish Neuroscience Institute, Swedish Medical Center, Seattle, Washington, USA
}

\begin{abstract}
Objectives: To review the relationship of the internal carotid artery, and carotid canal to the mandibular condyle, specifically from an infratemporal fossa approach. Skull base procedures which involve the middle cranial fossa utilize an infratemporal fossa approach either as the primary or adjunct surgical approach often performed with access osteotomies. In these surgeries, injury to the internal carotid artery and carotid canal may occur leading to many vascular complications ranging from internal carotid artery transection and thrombosis to embolism of distal communicating segments. Hence, knowing the relationship of these important structures is of utmost importance for skull base surgeons. In addition, the necessity for this knowledge is critical for clinicians to be able to understand the mechanism by which medial displacement of the mandibular condyle may cause blunt internal carotid artery injury in the evaluation of trauma patients. Identification of these structures and understanding their relationship on imaging may be used in the decision process to perform angiography based imaging.

Methods: Twenty dry skulls were utilized for a total of forty sides and the distance between the proximal carotid canal and the medial aspect of the mandibular condyle was measured.

Results: The average distance between the mandibular condyle and the carotid canal on right and left sided specimens was 1.03 $\mathrm{cm}$ and $1.11 \mathrm{~cm}$, respectively. The length ranged from $0.2 \mathrm{~cm}$ to $1.7 \mathrm{~cm}$. No significant differences were found between right and left sides.

Conclusion: A clear understanding of the anatomical relationship between the carotid canal and the head of the mandible, an easily identifiable landmark, is important for clinicians and surgeons alike. A substantial distance variability was observed in the samples studied. The understanding of this relationship should help identify patients at risk for ICA injury during surgical approaches and in the trauma setting.
\end{abstract}

Keywords: anatomy; infratemporal fossa; neurosurgery; skull base; vascular injury

Anatomy 2016;10(3):193-199 @2016 Turkish Society of Anatomy and Clinical Anatomy (TSACA)

\section{Introduction}

Vascular relationships are important in relation to the skull base (Figure 1). Infratemporal approaches are often challenging and made more hazardous by the disruption of anatomy due to tumor displacement and invasion. Approaches involving the infratemporal fossa (ITF) may cause injury to the internal carotid artery (ICA) leading to the need for repair, ligation and/or sacrifice. Anatomical knowledge of this relationship is of upmost importance. $^{[1]}$

The ICA is one of the terminal branches of the common carotid artery, which frequently originates at the C3-C5 vertebrae where the common carotid artery bifurcates into the internal and external branches. ${ }^{[2]}$ 


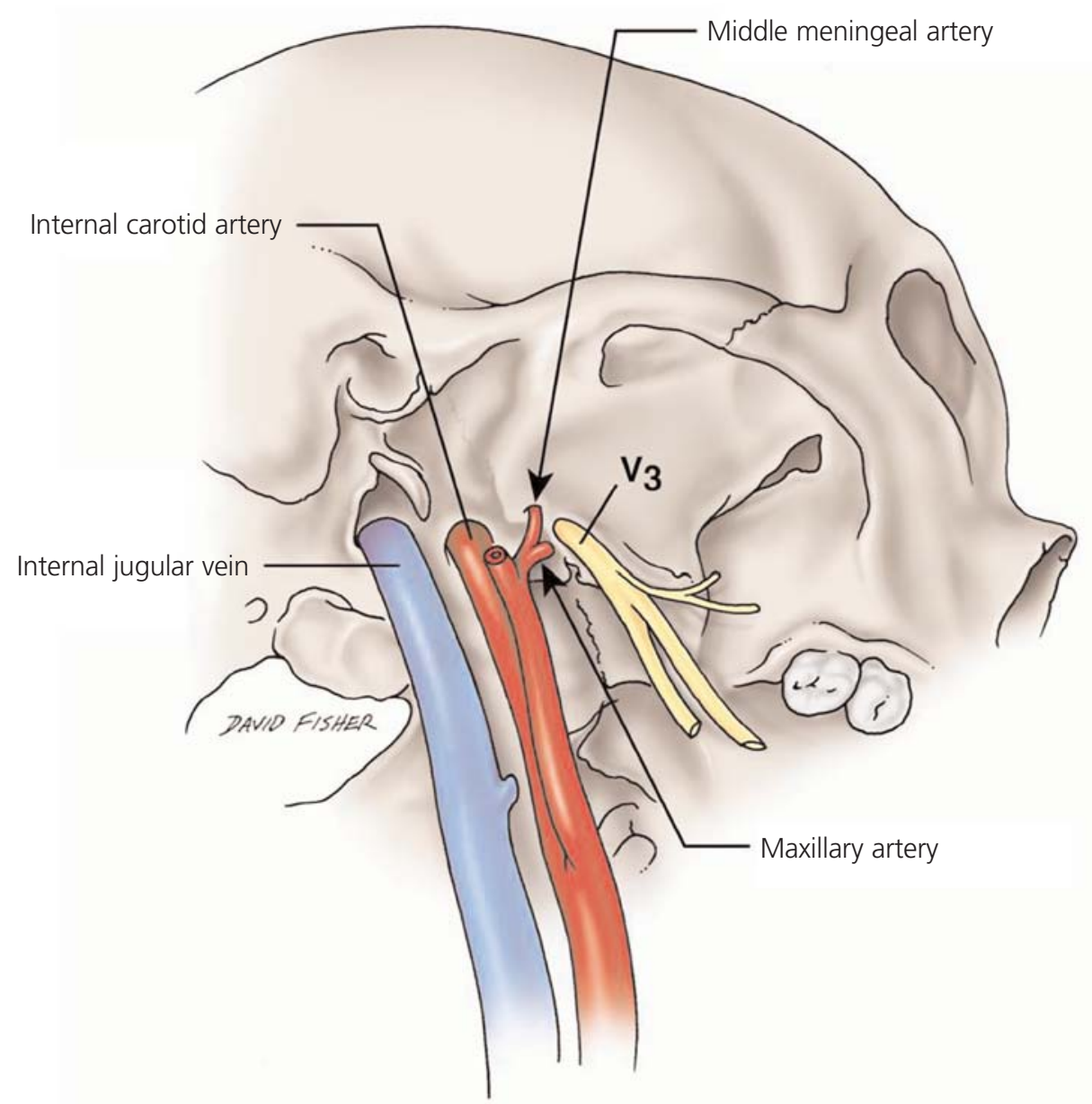

Figure 1. Schematic drawing of the vascular relationships at the skull base. Note the relationship between the medial edge of the mandibular fossa and the internal carotid artery as it enters the carotid canal. [Color figure can be viewed in the online issue, which is available at www.anatomy.org.tr]

Bouthillier et al. ${ }^{[3]}$ created a new classification system of the ICA using a numerical scale in the direction of blood flow, and described its segments according to a detailed understanding of the anatomical structures surrounding it and the compartments through which it travels. Commonly used today, it comprises seven segments: $\mathrm{C} 1$, cervical; $\mathrm{C} 2$, petrous; $\mathrm{C} 3$, lacerum; $\mathrm{C} 4$, cavernous; C5, clinoid; C6, ophthalmic; and C7, communicating. ${ }^{[3]}$

The cervical segment of the ICA begins at the level of the common carotid artery bifurcation. This segment is found inside the carotid sheath with the internal jugular vein lateral to the artery, and the vagus nerve usually in a posterolateral course. ${ }^{[1]}$ Inside the carotid sheath, the ICA is surrounded by areolar tissue containing fat, a venous plexus, and postganglionic sympathetic nerves. Rostrally, as the ICA enters the carotid canal, the carotid sheath divides into two layers. The inner layer continues as the periosteum of the carotid canal, and the outer layer continues as the periosteum of the extracranial sur- face (exocranium) of the skull base. The cervical segment of the ICA ends when it enters the carotid canal of the petrous bone anterior to the jugular foramen. ${ }^{[3]}$ The ICA does not branch prior to entering the carotid canal. The carotid canal, which encompasses the petrous ICA, terminates at the vertical line of the posterolateral margin of foramen lacerum.

As the carotid artery traverses the canal its direction is dependent upon the windings of its passage. ${ }^{[4]}$ The entrance of the carotid canal is bordered anterolaterally by a bony lamina that is part of the tympanic bone called the vaginal process. ${ }^{[3]}$ The periosteal lining of the carotid canal is densely adherent to a fibrocartilaginous ring at the entrance of the carotid canal. ${ }^{[5]}$ The opening of the carotid canal is most often oval. ${ }^{[6]}$ Its long axis shows a mediorostral to laterodorsal orientation with its largest diameter being $3-4.5 \mathrm{~mm}$ in newborns, $4-8 \mathrm{~mm}$ in two year olds, $6-7 \mathrm{~mm}$ in adolescents and adults on the right side and 6-8 $\mathrm{mm}$ on the left. ${ }^{[6]}$ 
As the artery exits from the internal orifice of the canal and ascends into the cranium, it encounters the body of the sphenoid bone. It is reflected ventrally and inferiorly through the cavernous sinus. It then rotates dorsally, beneath the anterior clinoid process, projecting superiorly and posteriorly, giving rise to the clinoid, ophthalmic and ultimately the communicating branches.

The first portion of the carotid canal is directed superiorly and anteriorly; its angle to the Frankfurt horizontal plane was measured as 113-1350 in newborns, 1101300 in 5-year olds, and 86-1100 in adults. ${ }^{[6]}$ The length of the ascending portion of the canal was $3.5-4.5 \mathrm{~mm}$ in newborns, $5.7 \mathrm{~mm}$ in 1-year olds, $7.3 \mathrm{~mm}$ in 3 -year-olds, $10 \mathrm{~mm}$ in 15 -year olds and $6.5-13.5 \mathrm{~mm}$ in adults. ${ }^{[6]}$

The aim of this study was to measure the distance between the medial edge of the mandibular condyle and the proximal carotid canal to review the relationship of the internal carotid artery, and carotid canal to the mandibular condyle, specifically from an ITF approach.

\section{Materials and Methods}

Twenty adult dry skulls were utilized for a total of forty sides and the distance between the proximal carotid canal at its lateral edge and the medial aspect of the mandibular condyle was measured (Figures $\mathbf{2}$ and 3). Specimens were derived from 12 males and 8 females. Measurements were made with microcalipers and were taken three times each and the average taken and recorded. Three of the authors (RIT, PAI, RST) made all the measurements. Photographs were taken of the specimens to document each skull's unique anatomy. Statistical analysis was performed using Statistica software (StatSoft, Inc., Gaithersburg, MD, USA). Statistical significance was set at $\mathrm{p}<0.05$.

\section{Results}

The average distance between the medial edge of the mandibular condyle and the lateral edge of the proximal carotid canal on right and left sided sides was $1.03 \mathrm{~cm}$ and $1.11 \mathrm{~cm}$, respectively. The length ranged from $0.2 \mathrm{~cm}$ to $1.7 \mathrm{~cm}$. No significant differences were found between right and left sides or sex. No specimen was found to have signs of temporomandibular joint disease or dislocation. None of the specimens were found to have damage or anomalies of the skull base near the regions studied.

\section{Discussion}

\section{Anatomy of the infratemporal fossa}

The ITF is defined as the area under the floor of the middle cranial fossa. ${ }^{[7]}$ It gives passage to several major cerebral vessels and cranial nerves. ${ }^{[7]}$ Knowledge of the surgical

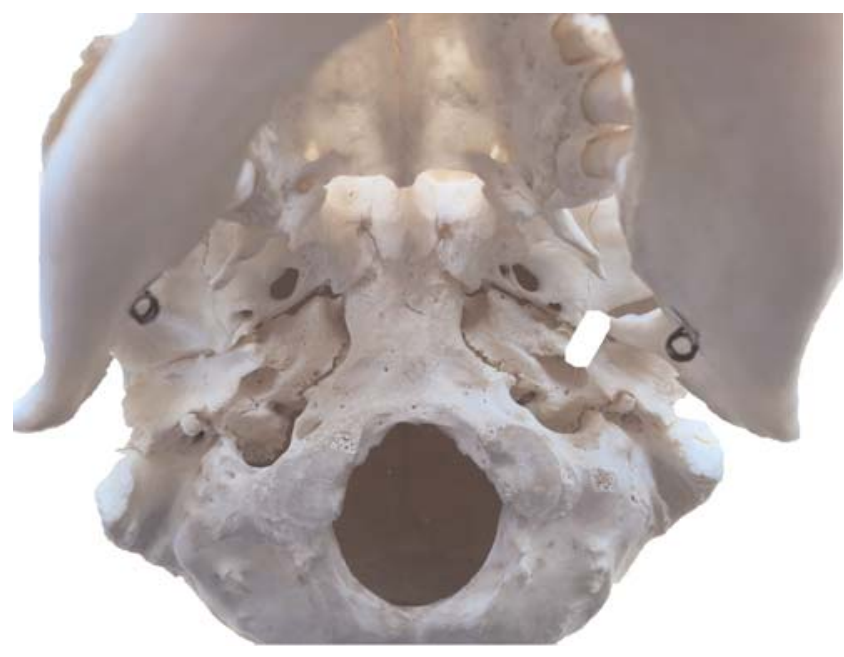

Figure 2. The skull base and the relationship between the medial edge of the mandibular head and the beginning of the carotid canal. [Color figure can be viewed in the online issue, which is available at wnw.anatomy. org.tr]

anatomy of the ITF is important to safely expose pathology of the parapharyngeal space, the distal cervical ICA, the extracranial course of cranial nerves VII through XII, and the lateral cranial base. Although a variety of surgical approaches to this region have been developed, the surgical anatomy is complex and remains challenging, often made more difficult by tumors that encase or displace key anatomical structures, increasing the complexity of surgical approaches. ${ }^{[7]}$

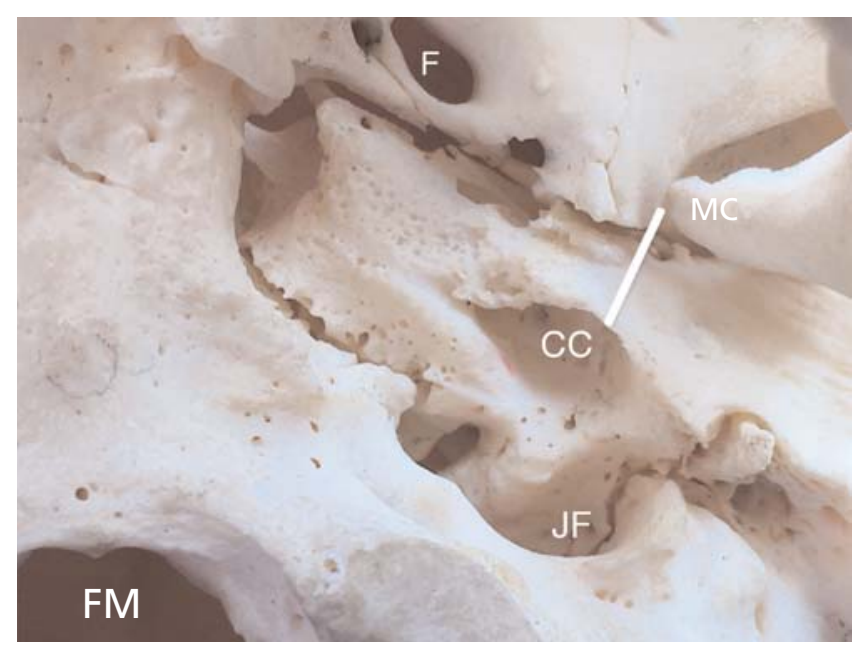

Figure 3. The skull base and the relationship between the medial edge of the mandibular head and the beginning of the carotid canal. Note the mandibular condyle (MC), opening of the proximal carotid canal (CC), jugular foramen (JF), foramen ovale (F), and foramen magnum (FM). The distance between $\mathrm{MC}$ and $\mathrm{CC}$ is seen as the white line. [Color figure can be viewed in the online issue, which is available at www.anatomy. org.tr] 
Bejjani et al. ${ }^{[7]}$ determined direct foraminal measurements of dried human skulls and noted the relationship of these foramina to each other and various landmarks. The anatomic relationships of muscles, nerves, arteries, and veins were carefully recorded, with special emphasis regarding the relationship of these structures to the styloid diaphragm. ${ }^{[7]}$

The styloid diaphragm is a key element in understanding the surgical anatomy of the ITF. It is oriented inferiorly and anteriorly being attached to the exocranial surface of the base of the cranium along a line from the anterior border of the mastoid process, through the styloid and vaginal (tympanic bone) processes, ending at the anterior border of the carotid canal.

The styloid diaphragm divides the ITF into the prestyloid region and the retrostyloid region. The prestyloid region contains the parotid gland and associated structures, including the facial nerve and external carotid artery. The retrostyloid region contains major vascular structures (ICA, internal jugular vein) and the initial exocranial portion of the lower cranial nerves IX through XII. ${ }^{[7]}$

If the mandible is laterally retracted and the medial pterygoid muscle is detached, the infratemporal space can be reached. In order to dislocate and mobilize the mandibular condyle, the attachment of the stylomandibular and sphenomandibular ligaments to the mandible are divided, dislocating the temporomandibular joint anteriorly. If more space is necessary, the condyle may be resected..$^{[2,5]}$

Using landmarks for the exocranial portion of the lower cranial nerves is useful in identifying them and avoiding injury during approaches to the high cervical ICA, the upper cervical spine, and the ITF. ${ }^{[7]}$ Its inferior boundary is the horizontal plane passing through the inferior border of the angle of the mandible and the roof, the infratemporal surface of the greater sphenoid wing and the exocranial surface of the petrotympanic region. The medial boundary is the lateral wall of the pharynx and the skin covering the parotid gland and the masseter. ${ }^{[7,8]}$

Anteriorly, it is limited by the maxillary tuberosity, the pterygomandibular raphe, and the muscles attached to it. Posteriorly, it is limited by the cervical prevertebral fascia and the underlying muscles. ${ }^{[8,9]}$

\section{Identification of the ICA within the surgical field}

A comprehensive knowledge of the anatomical relationship of the infrapetrous ICA and its adjacent structures is crucial (Figure 4). ${ }^{[9]}$ It is often difficult in cases of pathology to recognize these structures within a surgical field. ${ }^{[10]}$

Tumors of the skull base with carotid artery involvement either require carotid ligation, subtotal resection or have been deemed inoperable. ${ }^{[11]}$ Due to its course in the temporal bone, ICA can restrict surgical procedures for lesions extending to the middle skull base and ITF. Preservation of the ICA is demanded and techniques have been developed in order to assist the surgeon in its conservation. ${ }^{[2,12-18]}$

The Matas test (percutaneous compression of the carotid artery) and its newer variations have assisted the surgeon in the evaluation of carotid artery sacrifice and the need for bypass. First described in 1911 by Rudolph Matas, if percutaneous compression of the carotid artery produced neurological signs then it was inferred that the patient would not tolerate complete surgical occlusion. ${ }^{[19,20]}$ However, the technical difficulty of producing a prolonged complete occlusion of the carotid artery by percutaneous compression is considerable and failure to produce neurologic signs by compression, could not guarantee that the patient would tolerate complete surgical occlusion. This test has greatly been modified by contemporary endovascular techniques to be safer and

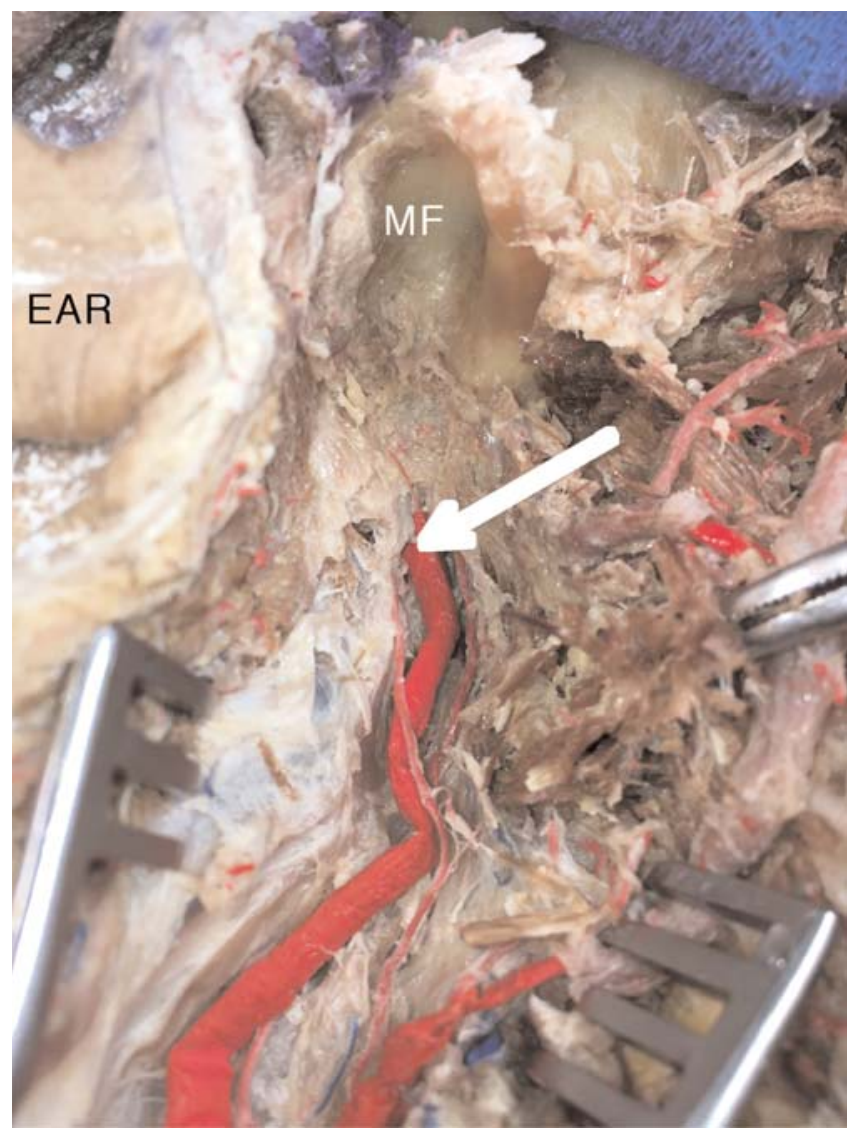

Figure 4. Cadaveric view of a trans-TMJ approach to the skull base. Note the intimate relationship between the medial edge of the mandibular fossa (MF) and the distal ICA at the skull base. [Color figure can be viewed in the online issue, which is available at www.anatomy. org.tr] 
more reliable, being largely replaced by balloon test occlusion. ICA ligation can lead to complications due to inadequate circulation in the acute period with subsequent infarction. Ligation of the ICA carries a high risk in patients over 50 years and should not be undertaken unless as an emergency. Even in younger patients, careful preoperative study with bilateral carotid angiography is needed. ${ }^{[19]}$

The absence of symptom development during a balloon test occlusion does not guarantee that a patient may become subsequently symptomatic after carotid artery sacrifice. ${ }^{[21]}$ Non-acute complications of carotid artery sacrifice include thrombosis and emboli of its distal branches. If the posterior communicating artery is large, this may rarely result in infarction of the posterior cerebral artery. ${ }^{[19]}$ However, performing a BTO can eliminate the need for a high flow radial artery or saphenous venous bypass and allow the clinician to perform a low flow STA-MCA bypass. ${ }^{[2]}$

As an alternative to carotid artery ligation, Urken et al. ${ }^{[1]]}$ proposed carotid artery bypass using autogenous saphenous vein graft with distal anastomosis to the infratemporal portion of the ICA. This procedure has been used for malignant skull base neoplasms removed en bloc with sacrifice of a portion of the ICA. ${ }^{[1]]}$

Sekhar demonstrated usage of a subtemporal-preauricular ITF approach to remove neoplasms involving the lateral and posterior cranial base. This approach has been found useful for the removal of neoplasms involving the clivus, sphenoidal area, petrous apex, orbit, cavernous sinus, middle fossa, infratemporaral fossa and the retro- and parapharyngeal areas. However, complications may arise due to ICA thrombosis and middle cerebral artery injury. ${ }^{[5]}$

In an attempt to prevent complications associated with ICA injury or ligation. Kawakami et al ${ }^{[12]}$ proposed performing radical surgery of malignant tumor occupying the ITF with en bloc resections of the tumor. This was achieved with preservation of the ICA. A substantial operative space was secured during surgery after early identification and opening of the carotid canal. In opening the canal, the carotid triangle can be first identified then shaved and cut as far as the spine of the sphenoid, allowing visualization of the tumor and the ICA. This permits en-bloc resection of the tumor with preservation of the ICA. ${ }^{[12]}$

With the advent and refinement of endovascular techniques, carotid sacrifice and subsequent bypass procedures may be avoided. Severe encasement of the ICA, a major factor in achieving gross total resection, may lead to arterial rupture. Stenting can be used to reinforce the cervical and infratemporal portions of the ICA with good success. ${ }^{[23]}$

The ITF approaches to the skull base were first introduced by Fisch for various lesions situated in the infralabyrinthine and apical compartments of the petrous pyramid and surrounding portions of the skull base. The aim of the type B and type C ITF approaches is to have control of the ICA. ${ }^{[9,24]}$ In ITF approaches type B and type $\mathrm{C}$, the main landmarks used during manipulation of the ICA are the basal turn of the cochlea, Eustachian tube, foramen spinosum, mandibular nerve and anterior foramen lacerum. ${ }^{[9,25]}$

Talebzadeh et al. ${ }^{[26]}$ measured a transverse distance average of $37.5 \mathrm{~mm}$ from the carotid artery to the zygomatic arch. This ranged from $29 \mathrm{~mm}$ to $48 \mathrm{~mm}$. The most medial aspect of the temporomandibular joint is the most lateral aspect of the ITF. In a study performed by Aslan et al. ${ }^{[9]}$ to clarify the anatomic relationship between surgical landmarks in type $b$ and type c ITF approach, the cochleariform process (CP) was used as their landmark for surgical orientation. The mean distance of the CP to the ICA genus was $9.2 \pm 1.6 \mathrm{~mm}$, while the mean distance from the cochlea to the ICA was $1.6 \pm 0.9 \mathrm{~mm} \cdot{ }^{[9]}$ Mason utilized an endoscopic approach and CT imaging, to find a mean distance to the petrous ICA of $16.34 \mathrm{~mm}$ from the foramen rotundum, $4.88 \mathrm{~mm}$ from the ovale, and $5.11 \mathrm{~mm}$ from the spinosum in males. For females, the values were $16.4 \mathrm{~mm}$ from the rotundum and $4.36 \mathrm{~mm}$ each from the ovale and spinosum. ${ }^{[14]}$ Additionally, their study made observations as to the existence and locations of any dehiscence of the carotid canal. Carotid canal dehiscence localization is of extreme importance during surgery with a frequency of approximately $20 \%$. The primary location of dehiscence was found to be the superior wall of the horizontal portion of the carotid canal. Dehiscence of the vertical portion is extremely rare. ${ }^{[9]}$ Lawton and Spetzler ${ }^{[27]}$ estimate the risk of ICA rupture by tumor infiltration to be approximately $18 \%$, exceeding the risk of revascularization procedures.

In a study of 605 facial trauma patients with mandibular condyle fractures by Vranis, it was found that 5.5 percent of patients had concomitant ICA injury. Severe vascular injury, defined as a Biffl grade greater than one, was present in 6 percent of extracapsular mandibular condyle fractures. ${ }^{[28]}$ Injury to the ICA can occur by several mechanisms including medial dislocation of the condylar head with impingement of the carotid artery, medial dislocation of the inferior fragment of the condylar fracture, posterior translation of the ramus as it may compress the ICA against the verte- 
bral transverse process and lateral displacement of the fracture if it allows the ramus of the mandible to migrate superiorly and medially. ${ }^{[28]}$ This emphasizes the need for the recognition of the proximity of the ICA to the mandibular condyle as it exceeds its surgical use and should be suspected in the evaluation of patients with facial trauma.

Our present study found the average lengths between the mandibular fossa and the carotid canal on right and left sided specimens were $1.03 \mathrm{~cm}$ and $1.11 \mathrm{~cm}$, respectively. The length ranged from $0.2 \mathrm{~cm}$ to $1.7 \mathrm{~cm}$. It is an important landmark as this area is simple to identify and often encountered during access osteotomies. ${ }^{[1,29-31]}$

\section{Conclusion}

An anatomical understanding of the relationship of the carotid canal, which encases only a segment of the ICA, is of immense importance for the surgeon. Special care and a strong anatomical knowledge are needed to prevent complications such as ICA laceration, thrombosis and subsequent emboli. This is more critical when there is invasion or dehiscence of the ICA by tumor pathology. We describe the approximate distance between the most medial border of the mandibular fossa, an easily identifiable landmark during mandibulotomy or dislocation of the mandibular condyle, and the carotid canal. Preoperative identification through imaging is critical as our series revealed significant variation among skull samples.

\section{References}

1. Sanna M, Shin SH, Piazza P, Pasanisi E, Vitullo F, Di Lella F, Bacciu A. Infratemporal fossa approach type a with transcondylar-transtubercular extension for Fisch type C2 to C4 tympanojugular paragangliomas. Head Neck 2014;36:1581-8.

2. Sekhar LN, Schramm VL Jr, Jones NF, Yonas H, Horton J, Latchaw RE, Curtin H. Operative exposure and management of the petrous and upper cervical internal carotid artery. Neurosurgery 1986;19: 967-82.

3. Bouthillier A, van Loveren HR, Keller JT. Segments of the internal carotid artery: a new classification. Neurosurgery 1996;38:425-33.

4. Smith NR. Surgical anatomy of the arteries: with plates and illustrations. Baltimore (MD): J. N. Toy and W. R. Lucas; 1835. p. 61-2.

5. Sekhar LN, Schramm VL, Jones NF. Subtemporal- preauricular infratemporal fossa approach to large lateral and posterior cranial base neoplasms. J Neurosurg 1987;67:488-99.

6. Lang J. Skull base and related structures: atlas of clinical anatomy. 2nd ed. Stuttgart: Schattauer; 1996. p. 60-180.

7. Bejjani GK, Sullivan B, Salas-Lopez E, Abello J, Wright DC, Jurjus A, Sekhar LN. Surgical anatomy of the infratemporal fossa: the styloid diaphragm revisited. Neurosurgery 1998;43:842-52; discussion $852-3$.
8. Fisch U, Mattox D. Microsurgery of the skull base. New York (NY): Thieme Medical Publishers; 1988. p. 286-301.

9. Aslan A, Balyan FR, Taibah A, Sanna M. Anatomic relationships between surgical landmarks in type $\mathrm{b}$ and type $\mathrm{c}$ infratemporal fossa approaches. Eur Arch Otorhinolaryngol 1998;255:259-64.

10. Guo YX, Sun ZP, Liu XJ, Bhandari K, Guo CB. Surgical safety distances in the infratemporal fossa: three-dimensional measurement study. Int J Oral Maxillofac Surg 2015;44:555-61.

11. Urken ML, Biller HF, Haimov M. Intratemporal carotid artery bypass in resection of base of skull tumor. Laryngoscope 1985;95:1472-7.

12. Kawakami K, Kawamoto K, Tsuji H. Opening of the carotid canal in the skull base surgery: drilling of the carotid canal triangle. No Shinkei Geka 1993;21:1013-9.

13. Catalano PJ, Bederson J, Turk JB, Sen C, Biller HF. New approach for operative management of vascular lesions of the infratemporal internal carotid artery. Am J Otol 1994;15:495-501.

14. Mason E, Gurrola J 2nd, Reyes C, Brown JJ, Figueroa R, Solares CA. Analysis of the petrous portion of the internal carotid artery: landmarks for an endoscopic endonasal approach. Laryngoscope 2014;124:1988-94.

15. Sabit I, Schaefer SD, Couldwell WT. Modified infratemporal fossa approach via lateral transantral maxillotomy: a microsurgical model. Surg Neurol 2002;58:21-31.

16. Leonetti JP, Benscoter BJ, Marzo SJ, Borrowdale RW, Pontikis GC. Preauricular infratemporal fossa approach for advanced malignant parotid tumors. Laryngoscope 2012;122:1949-53.

17. Froelich SC, Abdel Aziz KM, Levine NB, Pensak ML, Theodosopoulos PV, Keller JT. Exposure of the distal cervical segment of the internal carotid artery using the trans-spinosum corridor: cadaveric study of surgical anatomy. Neurosurgery 2008;62: ONS354-61; discussion ONS361-2.

18. Suhardja AS, Cusimano MD, Agur AM. Surgical exposure and resection of the vertical portion of the petrous internal carotid artery: anatomic study. Neurosurgery 2001;49:665-9; discussion 669-70.

19. Voris HC. Complications of ligation of the internal carotid artery. J Neurosurg 1951;8:119-31.

20. Wang H, Lanzino G, Kraus RR, Fraser KW. Provocative test occlusion or the matas test: who was rudolph matas? J Neurosurg 2003;98: 926-8.

21. Couldwell WT, Liu JK, Amini A, Kan P. Submandibular-infratemporal interpositional carotid artery bypass for cranial base tumors and giant aneurysms. Neurosurgery 2006;59:ONS353-9; discussion ONS359-60.

22. Almefty K, Spetzler RF. Management of giant internal carotid artery aneurysms. World Neurosurg 2014;82:40-2.

23. Sanna M, Piazza P, De Donato G, Menozzi R, Falcioni M. Combined endovascular-surgical management of the internal carotid artery in complex tympanojugular paragangliomas. Skull Base 2009; 19:26-42.

24. Fisch U, Pillsbury HC. Infratemporal fossa approach to lesions in the temporal bone and base of the skull. Arch Otolaryngol 1979;105: 99-107.

25. Liu J, Pinheiro-Neto CD, Fernandez-Miranda JC, Snyderman CH, Gardner PA, Hirsch BE, Wang E. Eustachian tube and internal carotid artery in skull base surgery: an anatomical study. Laryngoscope 2014;124:2655-64. 
26. Talebzadeh N, Rosenstein TP, Pogrel MA. Anatomy of the structures medial to the temporomandibular joint. Oral Surg Oral Med Oral Pathol Oral Radiol Endod 1999;88:674-8.

27. Lawton MT, Spetzler RF. Internal carotid artery sacrifice for radical resection of skull base tumors. Skull Base Surg 1996;6:119-23.

28. Vranis NM, Mundinger GS, Bellamy JL, Schultz BD, Banda A, Yang R, Dorafshar AH, Christy MR, Rodriguez ED. Extracapsular mandibular condyle fractures are associated with severe blunt internal carotid artery injury: analysis of 605 patients. Plast Reconstr Surg 2015;136:811-21
29. Maheshwari GU, Chauhan S, Kumar S, Krishnamoorthy S. Access osteotomy for infratemporal tumors: two case reports. Ann Maxillofac Surg 2012;2:77-81.

30. Ye ZX, Yang C, Chen MJ, Abdelrehem A. A novel approach to neoplasms medial to the condyle: a condylectomy with anterior displacement of the condyle. Int J Oral Maxillofac Surg 2016;45:427- 32.

31. Vilela MD, Rostomily RC. Temporomandibular joint-preserving preauricular subtemporal-infratemporal fossa approach: surgical technique and clinical application. Neurosurgery 2004;55:143-53; discussion 153-4.

Correspondence to: Joe Iwanaga, DDS, PhD

Seattle Science Foundation, 550 17th Ave, James Tower, Suite 600, Seattle, WA, 98122, USA

Phone: +1 20673265 00, Fax: +1 2067326599

e-mail: joei@seattlesciencefoundation.org

Conflict of interest statement: No conflicts declared.

This is an open access article distributed under the terms of the Creative Commons Attribution-NonCommercial-NoDerivs 3.0 Unported (CC BY-NC-ND3.0) Licence (http://creativecommons.org/licenses/by-nc-nd/3.0/) which permits unrestricted noncommercial use, distribution, and reproduction in any medium, provided the original work is properly cited. Please cite this article as: Alonso F, Bernard S, Irwin PA, Tubbs RI, Iwanaga J, Loukas M, Oskouian RJ, Tubbs RS. The relationship between the carotid canal and mandibular condyle: an anatomical study with application to surgical approaches to the skull base via the infratemporal fossa. Anatomy 2016;10(3):193-199. 